\title{
Proceso diagnóstico de las familias con un hijo/a con TEA
}

\section{Diagnostic process of the families with a child with ASD Syndrome}

\author{
Antía Cid Rodríguez, Silvia López Larrosa \\ Universidad de A Coruña
}

\begin{abstract}
Resumen
Este trabajo analiza el proceso diagnóstico en las familias cuyos hijos/as tienen Síndrome del Trastorno del Espectro Autista (TEA). El TEA no afecta solo de manera individual a una persona, sino que afecta a nivel familiar. Por ese motivo, se investiga de qué manera afrontan las familias el diagnóstico de este Síndrome, con qué recursos cuentan, qué tipo de información se les proporciona en el mismo momento del diagnóstico, a qué tipos de centros acuden en busca de éste, etc. Se contó con la participación de 10 familias de niños/as con un diagnóstico de TEA y se elaboró un cuestionario con preguntas abiertas y cerradas. A partir de ellas, se puede señalar que tras un tiempo de sospechas, las familias buscaron un diagnóstico, que, en la mayoría de los casos, les proporcionaron servicios privados. No obstante, el centro escolar al que acuden sus hijos es el principal apoyo con el que cuentan tras el diagnóstico.
\end{abstract}

Palabras clave: familia, discapacidad, Síndrome de TEA, diagnóstico.

\begin{abstract}
This paper analyses the diagnostic process families whose children have Autism Spectrum Disorder Syndrome (ASD) go through. ASD affects individually but also affects the whole family. For this reason, our aim is to investigate how families face diagnosis, what resources they have, what kind of information they get at the moment of diagnosis, what services they use, etc. Ten families with a child with ASDs participated. A questionnaire with open-ended and closed questions was developed. Results show that after a time of suspicion, the families search for a diagnosis, that in most cases, they are given by private services. However, their children's school is the main resource they can count on after diagnosis.
\end{abstract}

Keywords: family, disability, ASD Syndrome, diagnosis.

El TEA constituye un grupo de trastornos que pueden causar problemas significativos de socialización, comunicación y conducta. Las personas con TEA procesan la información de manera distinta a los demás. No obstante, el TEA no afecta solo de manera individual a una persona, sino que afecta a nivel familiar (van Steijn et al., 2015). El diagnóstico de una discapacidad sin duda afecta a los padres y al resto de la familia, genera preocupación, miedo, rabia (Gómez, 2010; Seligman y Darling, 2007) y evidencia la necesidad de que los padres reciban información, formación y apoyo (Muñoz, 2011). Por este motivo, se ha investigado de qué manera afrontan las familias el diagnóstico de este Síndrome, con qué recursos materiales o humanos cuentan, qué tipo de información se les proporciona en el mismo momento del diagnóstico, a qué tipos de centros acuden en busca de éste, etc. En concreto, dos son los objetivos de este estudio: 1. Detectar las necesidades de las familias con miembros con TEA a la hora del diagnóstico. 2. Analizar el impacto que tiene el diagnóstico de TEA en las familias.

\section{Método}

\section{Participantes}

Se contó con la participación de 10 familias de siete niños y tres niñas con un diagnóstico de TEA con edades comprendidas entre los 2 y los 13 años (media de edad $=7,8$ años, $D E=3,79)$. En todos los casos, contestaron las madres. En su mayoría tenían estudios universitarios (6 casos) o medios (3 casos). Solo una madre tenía estudios primarios. La mitad de los niños eran hijos únicos y la otra mitad tenía otro hermano (4 casos) o dos hermanos (1 caso). De los que tenían hermanos, en tres casos más de un hijo tenía TEA. La mayoría de las familias vivían en un contexto urbano ( 7 casos) y 3 vivían en un contexto no urbano.

\section{Materiales}

Se elaboró un cuestionario de 33 preguntas abiertas y cerradas. El cuestionario, elaborado específicamente para este estudio y denominado "Cuestionario de Necesidades formativas y sociales", recoge información sociodemográfica y sobre el proceso diagnóstico: cuándo y cómo se llegó a él, información proporcionada, ayudas obtenidas y propuestas de mejora de dicho proceso.

\section{Procedimiento}

En primer lugar, se escogieron dos centros que reunían las características de ser de reciente creación y que consideran como uno de sus pilares de actuación la atención individualizada, aunque uno de ellos parece más orientado a considerar a las familias (la asociación) teniendo en cuenta cómo se constituye. El procedimiento de recogida de datos fue el mismo. En ambos casos, se solicitó el permiso a la dirección. Una vez obtenido, se entregaron los cuestionarios a dos personas responsables de la directiva. Estas personas entregaron los cuestionarios a las familias para que los cubriesen y, cuando éstos ya estuvieron cubiertos, los devolvieron.

En el caso de la Asociación, se entregaron 10 
cuestionarios (ya que era el número de familias con las que contaban) pero devolvieron cubiertos 5 . Sin embargo, en el caso del Centro Psicopedagógico todos los entregados fueron cubiertos (5).

\section{Resultados}

Con respecto al primer objetivo, cuando se pregunta a las familias sobre la primera vez que buscaron ayuda, la mayoría contestan que acudieron a un servicio privado ( 6 casos). En dos casos acudieron a un servicio público y otros dos recurrieron a ambos, servicio público y privado.

En 7 casos, las familias fueron a un especialista (logopeda, psicólogo...) y los demás acudieron al pediatra. En esta búsqueda de un diagnóstico, la mayoría de las familias (8) no tuvieron que desplazarse para recibirlo, en contraposición a dos que tuvieron que ir a otra ciudad.

Con respecto a los comportamientos que despertaron sus sospechas, por ejemplo, la familia 1 contestó que su hijo mostraba: "Alta intolerancia a la frustración, conductas disruptivas en el aula, enfado permanente, disgrafía y aislamiento de sus compañeros".

La familia 7 en cambio destacó los siguientes comportamientos sobre sus hijos gemelos (uno diagnosticado de Asperger y otro de Autismo): "No hablaban, no soportaban ruidos ni cambios de rutinas, dejaron de comer".

El resto de las familias coinciden con las aportaciones anteriores y además añaden que sus hijos no miraban directamente a los ojos, tenían falta de afectividad con ellos en casa, falta de juego en el parque con otros niños, no señalaban, o imitaban, se alteraban mucho en clase, etc.

Por otro lado, las familias valoraron una serie de ítems sobre el proceso de diagnóstico (tiempo de espera, comunicación del diagnóstico, trato que recibieron y el asesoramiento recibido) según su experiencia personal usando una escala Likert de 1 (muy deficiente) a 4 (muy bueno). Lo que más valoraron fue el trato $(3,3)$, seguido de la comunicación y el asesoramiento (2,8 en ambos). Lo menos valorado fue el tiempo de espera $(2,7)$. No obstante, la importancia que le concedieron a cada una de las dimensiones difiere, de modo que lo que consideran más importante es el asesoramiento (10 sobre 10), seguido de la comunicación y el trato $(9,2$ sobre 10$)$ y lo menos, en este caso en consonancia con lo recibido realmente, es el tiempo de espera $(8,9$ sobre 10$)$. No obstante, los valores de importancia son elevados en todos los casos y la valoración de lo realmente recibido es algo más baja.

La mayor parte de las familias participantes (8) recibieron un informe con el diagnóstico por escrito. En dicho informe, a seis las informaron sobre necesidades médicas (especialistas a los que podría o debería acudir, medicamentos que debe tomar el niño, etc...) y necesidades educativas (centros que existen, apoyos que puede necesitar...) que tendrá su hijo. En cambio, al resto no les dieron esta información. Sobre las necesidades sociales (asociaciones, apoyo familiar...) informaron a cuatro familias. Sobre las necesidades materiales (organismos, bibliografía, etc...) solo informaron a una familia.

En cuanto a las ayudas recibidas, la escuela de sus hijos fue la más mencionada ( 9 casos), seguida por los médicos y la familia (5 casos) y las asociaciones (4 casos). Profundizando algo más, cuando se les preguntaba cómo les habían ayudado estas personas o entidades a afrontar el diagnóstico de su hijo/a y a trabajar con él en su día a día, podemos destacar la siguiente aportación de la familia 1: "Respetando su neurodiversidad y estimulando sus capacidades".

Los demás participantes contestaron que también les ayudaron dándoles su apoyo, mejorando la calidad de vida de sus familias en general, trabajando de forma individualizada con su hijo/a, promoviendo mediante el juego la interacción y comunicación verbal, informando de cómo reaccionar en distintas situaciones y cómo tratar al niño.

En cuanto al objetivo dos "Analizar el impacto que tiene el diagnóstico de TEA dentro de las familias", la familia número 5 señaló que un impacto a nivel social es que: "La gente no es comprensiva con lo que le pasa a nuestra niña, nos miran raro cuando ella se comporta de forma extraña. Dado que es tan pequeña no puede controlarse". No obstante, la vida en el interior de la familia también se ve afectada pues, como señala una madre, su vida gira en torno a su hijo: "'mi día empieza pensando en todas las actividades que tenemos que hacer en el día y en cómo va a ser su descanso nocturno". También se ven afectadas las rutinas pese a que en algún caso señalan: "hacemos vida normalizada, mi hijo está totalmente integrado en todos los ámbitos".

\section{Discusión}

De los resultados se desprende que es muy importante que la gente conozca lo que es el Síndrome de TEA, qué características tiene, porqué se comportan así y que se empiece a reconocer que son personas a las que les cuesta integrarse. En este aspecto la escuela juega un papel esencial, reconocido por los padres participantes, como apoyo para ellos y sus hijos, frente a los profesionales médicos. Pese a ello, la respuesta es mejorable. Como incide una madre: "En los centros educativos no existe información a los alumnos que tienen que convivir con el alumno con TEA. Tampoco existe una educación a nivel de la sociedad en general. Es dificil localizar centros que tengan actividades lúdicas y deportivas de apoyo".

Sin duda, el trato en la escuela tiene una duración temporal mucho mayor que el trato proporcionado por los servicios sanitarios. Además, la escuela puede jugar un importante papel en este proceso de normalización de la diferencia. Aspecto en el que incide una de las madres participantes: "La sensibilización puede conseguirse divulgando las cualidades positivas de los, las TEA. Poner en valor sus puntos favorables conseguirá su aceptación. Su aislamiento es peor que su Síndrome”.

Para finalizar, son evidentes el impacto que el diagnóstico de TEA tiene en la vida de las familias y en sus relaciones con el exterior, y el desconocimiento social con respecto al TEA. Las madres son capaces de valorar diversos elementos del proceso diagnóstico 
pdf.

incluso de manera positiva aun cuando la transmisión de información en el momento del diagnóstico fue insuficiente, tanto con respecto a las necesidades médicas y educativas como sociales. No obstante, con el tiempo parecen haber obtenido información, lo que se hace patente cuando se les ofrece la oportunidad de expresarse y demuestran el conocimiento profundo que al menos algunas de las familias participantes han alcanzado. Pero no es suficiente, también necesitan asesoramiento y apoyo. Y parece lógico si en el camino con su hijo se encuentran el rechazo de otros y la falta de coordinación de los profesionales, además, podemos suponer, de las dificultades inherentes al TEA.

\section{Referencias}

Gómez, B. (2010). Resilencia individual y familiar. Programa Gaztedi. País Vasco: Servicio Integral de Apoyo a Adolescentes.
Muñoz, A. (2011). Impacto de la discapacidad en las familias. Revista Digital Innovación y Experiencias Educativas, 38 (1), 1-8.

http://www.revistaeducacion.educacion.es/re356/ re356.

Seligman, M. y Darling, R.B. (2007). Ordinary families, special children. Nueva York: Guilford Press.

van Steijn, D.J.; Oerlemans, A.M.; van Aken, M. A. G.; Buitelaar, J.K. y Rommelse, N.N.J. (2015). The influence of parental and offspring autism spectrum disorder (ASD) and attention-deficit/hyperactivity disorder (ADHD) symptoms on family climate. Journal of Child and Family Studies, 24(7), 2021-2030. doi: 10.1007/s10826-014-0002-9. 